\title{
Performed Observation Result
}

National Cancer Institute

\section{Source}

National Cancer Institute. Performed Observation Result. NCI Thesaurus. Code C93421.

The finding obtained by observing, monitoring, measuring or otherwise qualitatively or quantitatively recording one or more aspects of physiologic or psychologic processes. 